\title{
Letter to the Editor: Specific and Stepwise Postoperative Rehabilitation Program Is Needed in the Elderly After Hip Fracture Surgery
}

With great interest, we have encountered the article by Jang et al. ${ }^{1)}$. We appreciate the effort of this study group to evaluate the effect of perioperative geriatric intervention on healthcare outcomes in patients undergoing surgery for hip fracture. This type of research is needed because of the lack of evidence-based perioperative multidisciplinary management and its cost-effectiveness analysis in Korea, although similar studies have already been published in Europe $^{2-4)}$. The topic of the study is also relevant in regard to the increasing rate of hip fracture and related growing financial burden to the healthcare system. Herein, we have some suggestions for the study.

Our main suggestion is regarding the components of geriatric intervention. In the study, the geriatric intervention consists of the following: initial screening and risk management for physical status, nutritional status, cognitive function, and mood status; prevention of malnutrition, polypharmacy, incontinence, and falls; rehabilitation in the geriatric ward; and discharge planning, including a long-term care plan. Although these components are sufficient for comprehensive geriatric care, the program does not have any specialized intervention related to the postoperative hip joint status. The target participants are not only the elderly but also patients with hip problems. Therefore, a more specific and stepwise postoperative rehabilitation program should be included as part of the geriatric intervention.

Initiation of an integrated rehabilitation program in the early phase following hip fracture surgery is essential in elderly patients. Over the past few years, we have been trying to establish a multidisciplinary fragility fracture care (particularly for hip fracture) project with several orthopedic surgeons and a geriatrician in Korea. This year, it has launched as a nationwide multicenter project for fracture liaison service, which was funded by the Ministry of Health and Welfare. The purpose of this study was to develop a standardized fracture-integrated rehabilitation management of hip joint
(FIRM-HIP) in the elderly, based on the critical rehabilitation pathway for fragility fractures in both hospital and community settings. From August to October, 2016, a preliminary study of FIRM-HIP has been conducted in two rehabilitation centers (Seoul National University Bundang Hospital and Chung-Ang University Hospital).

Forty-seven consecutive elderly patients with hip fracture were enrolled (mean age, 81.4 years). All patients received FIRM-HIP care, which consisted of physical therapy (lower limb strengthening and gait training using assistive device, twice a day), occupational therapy (3 times in hospital course), fall prevention, and discharge planning for 2 weeks (10 days) after hip surgery. No serious adverse events were reported among the patients who received FIRM-HIP care. On an average of 6.3 days postsurgery, patients were transferred to the department of rehabilitation medicine. After FIRM-HIP care, mobility scores, activities of daily living, quality of life scores, and frailty scores were significantly improved.

From this pilot study, we can develop and standardize the FIRM-HIP program and plan to conduct a controlled trial that compares FIRM-HIP care to conventional postoperative management. Furthermore, a rehabilitation program, including advanced techniques such as lower-body positive pressure treadmill training, has also been implemented. Specific and stepwise postoperative rehabilitation programs can be established through these studies.

Conflicts of Interest Disclosures: The researchers claim no conflicts of interest.

\section{Acknowledgments}

This research was supported by a grant of the Korea Health Technology R\&D Project through the Korea Health Industry Development Institute (KHIDI), funded by the Ministry of Health \& Welfare, Republic of Korea (grant number: HC15C1234). 


\section{Sang Yoon Lee ${ }^{1}$, Jaewon Beom ${ }^{2}$}

${ }^{1}$ Department of Physical Medicine \& Rehabilitation, Chung-Ang University Hospital, Seoul, ${ }^{2}$ Department of Rehabilitation Medicine, Chungnam National University Hospital, Daejeon, Korea

Corresponding Author: Jaewon Beom, $\mathrm{MD}, \mathrm{PhD}$

Department of Rehabilitation Medicine, Chungnam National

University Hospital, 282 Munhwa-ro, Jung-gu, Daejeon 35015, Korea

Tel: +82-42-338-2418, Fax: +82-42-338-2461

E-mail: powe5@naver.com

Received: November 29, 2016

Revised: November 30, 2016

Accepted: December 4, 2016

\section{REFERENCES}

1. Jang IY, Lee YS, Jung HW, Chang JS, Kim JJ, Kim HJ, et al. Clinical outcomes of perioperative geriatric intervention in the elderly undergoing hip fracture surgery. Ann Geriatr Med Res 2016;20:125-30.

2. Karlsson Å, Berggren M, Gustafson Y, Olofsson B, Lindelöf N, Stenvall M. Effects of geriatric interdisciplinary home rehabilitation on walking ability and length of hospital stay after hip fracture: a randomized controlled trial. J Am Med Dir Assoc 2016; 17:464.e9-464.e15.

3. Lahtinen A, Leppilahti J, Harmainen S, Sipilä J, Antikainen R, Seppänen ML, et al. Geriatric and physically oriented rehabilitation improves the ability of independent living and physical rehabilitation reduces mortality: a randomised comparison of 538 patients. Clin Rehabil 2015;29:892-906.

4. Prestmo A, Hagen G, Sletvold O, Helbostad JL, Thingstad P, Taraldsen K, et al. Comprehensive geriatric care for patients with hip fractures: a prospective, randomised, controlled trial. Lancet 2015;385:1623-33. 